\title{
Generalized Pairwise Z-Complementary Codes
}

\author{
Lifang Feng, Pingzhi Fan, Senior Member, IEEE, Xiaohu Tang, Member, IEEE, and Kok-keong Loo, Member, IEEE
}

\begin{abstract}
An approach to generate generalized pairwise Z-complementary (GPZ) codes, which works in pairs in order to offer a zero correlation zone $(\mathrm{ZCZ})$ in the vicinity of zero phase shift and fit extremely well in power efficient quadrature carrier modems, is introduced in this letter. Each GPZ code has MK sequences, each of length $4 \mathrm{NK}$, where $\mathrm{M}$ is the number of $\mathrm{Z}$-complementary mates, $\mathrm{K}$ is a factor to perform Walsh-Hadamard expansions, and $\mathrm{N}$ is the sequence length of the $Z$-complementary code. The proposed GPZ codes include the generalized pairwise complementary (GPC) codes as special cases.
\end{abstract}

Index Terms-Generalized pairwise complementary codes, Z-complementary codes, zero correlation zone.

\section{INTRODUCTION}

$\mathbf{I}$ $\mathrm{N}$ general, there are two types of code-division multiple access (CDMA) codes. One is unitary code, which works on a one-code-per-user basis, including orthogonal codes such as Walsh-Hadamard sequences [1], orthogonal variable spreading factor (OVSF) codes, pseudorandom codes, zero correlation zone (ZCZ) codes [2], [3], etc.; the other is complementary code, which was first studied by Golay [4] in the early 1960s for their possible applications in radar systems. The complementary codes work on one-flock-per-user basis. It is noted that if the flock size is limited to two, complementary codes become pairwise complementary codes.

It has been shown in [5] that a unitary code set can never give ideal cross-correlation and autocorrelation properties. In other words, interference-free windows (IFWs) or ZCZ for any unitary codes will never be equal to its code length. Other works have also been reported on the design of a unitary CDMA code set with a ZCZ [2], [3]. However, these ZCZ codes can offer a relatively small $\mathrm{ZCZ}$, let alone the fact that most of them are non-binary codes, which make it extremely difficult to be used in a real system. Generalized pairwise complementary (GPC) codes were proposed in [6] based on complete complementary codes and generalized even shift orthogonal (GESO) sequences [7]. However, the binary complete complementary sets are limited by the length (i.e., $2^{a} 10^{b} 26^{c}$, where $a, b, c$ are nonnegative

Manuscript received October 22, 2007; revised January 16, 2008. This work was supported in part by the National Science Foundation of China (NSFC) under Grant 60772087, in part by the 111 Project under Grant 111-2-14, in part by the National 863 High-Tech R\&D Program under Grant 2007AA01Z228, and in part by the Foundation for the Author of National Excellent Doctoral Dissertation of PR China (FANEDD) under Grant 200341. The associate editor coordinating the review of this manuscript and approving it for publication was Prof. Zhengdao Wang.

L. Feng, P. Fan, and X. Tang are with the Provincial Key Lab of Information Coding and Transmission, Institute of Mobile Communications, Southwest Jiaotong University, Chengdu, Sichuan 610031, China (e-mail: lifang.feng@gmail.com; p.fan@ieee.org; xhutang@ieee.org).

K.-K. Loo is with the School of Engineering and Design, Brunel University, London UB8 3PH, UK (e-mail: Jonathan.Loo@brunel.ac.uk).

Digital Object Identifier 10.1109/LSP.2008.919997 integers) which limits the length of GPC codes. Furthermore, for a given complete complementary set containing $P$ sequences, it has been shown that there exist only maximally $P$ complementary mates which limits the number of GPC codes. Families of sequence pair with ZCZ were also proposed in [8], while their implementation in practical systems is complicated.

This letter tries to combine the advantage of unitary codes (implementation simplicity) and Z-complementary codes (fewer restrictions are on the length of complementary sequences and the number of complementary mates) [9]. A generation approach based on Z-complementary codes [9] and generalized even shift orthogonal like (GESO-like) sequences which is extended by GESO sequences [7] is proposed to construct generalized pairwise Z-complementary (GPZ) codes. The GPZ codes work in pairs, allowing the use of extremely power-efficient quadrature phase-shift keying (QPSK) modulation.

The rest of this letter is outlined as follows. Section II introduces Z-complementary codes. Section III describes how to construct a GESO-like sequence set, which is the basis of GPZ codes. Section IV explains how to use Walsh-Hadamard matrix expansion to increase the number of sequences in a GESO-like sequence set and describes the odd shift correlation elimination algorithm to remove nonzero odd-shift correlation levels to obtain the GPZ codes. The correlation properties of the GPZ codes will be given in Section V, followed by the conclusions.

\section{PRELIMINARIES}

Let $\boldsymbol{a}=(a(0), a(1), \ldots, a(N-1))$ and $\boldsymbol{b}=(b(0), b(1), \ldots, b(N-1))$ denote two complex-valued sequences of length $N$. Let $x^{*}$ denote the complex conjugate of $x$, and subscript addition $i+\tau$ in (2) is performed modulo $N$.

The aperiodic and periodic correlation functions between $\boldsymbol{a}$ and $\boldsymbol{b}$ are given as follows:

$$
A_{a, b}(\tau)= \begin{cases}\sum_{i=0}^{N-1-\tau} a(i) b(i+\tau)^{*}, & 0 \leq \tau<N \\ \sum_{i=0}^{N-1+\tau} a(i-\tau) b(i)^{*}, & 1-N \leq \tau<0 \\ 0, & |\tau| \geq N\end{cases}
$$

and

$$
P_{a, b}(\tau)=\sum_{i=0}^{N-1} a(i) b(i+\tau)^{*}, \quad 0 \leq \tau<N .
$$

In particular, the correlation function is called auto-correlation function (ACF) if $a=b$, or cross-correlation function (CCF) otherwise. 
A set of $P$ binary sequences $\left\{\boldsymbol{a}_{1}, \boldsymbol{a}_{2} \ldots \boldsymbol{a}_{P}\right\}$, each having length $N$, is called a set of Z-complementary sets $\left(\mathrm{Z}-\mathrm{CS}_{P}^{N}\right)$ if

$$
\sum_{i=1}^{P} A_{a_{i}, a_{i}}(\tau)= \begin{cases}\sum_{i=1}^{P} E_{a_{i}}, & \tau=0 \\ 0, & 1 \leq \tau \leq Z-1\end{cases}
$$

where $Z$ is called ZCZ and $E_{a_{i}}$ is the energy of sequence $a_{i}$.

Obviously, the definition of Z-complementary sets includes the conventional binary complementary set as a special case when $Z=N$. Besides, another set of $Z-\mathrm{CS}_{P}^{N}\left\{\boldsymbol{b}_{1}, \boldsymbol{b}_{2}, \ldots, \boldsymbol{b}_{P}\right\}$ is called a Z-complementary mate of $\mathrm{Z}-\mathrm{CS}_{P}^{N}\left\{\boldsymbol{a}_{1}, \boldsymbol{a}_{2}, \ldots, \boldsymbol{a}_{P}\right\}$ if

$$
\sum_{i=1}^{P} A_{a_{i}, b_{i}}(\tau)=0, \quad 0 \leq \tau \leq Z-1 .
$$

When $Z=N$, the Z-complementary mate becomes the conventional binary complementary mate which is unique once $\left\{\boldsymbol{a}_{1}, \boldsymbol{a}_{2}, \ldots, \boldsymbol{a}_{P}\right\}$ is fixed. When $Z<N$, usually there is more than one mate.

\section{Generalized Even Shift Orthogonal-Like SEQUENCES}

Let us consider a set $S=\left\{S^{1}, S^{2}, \ldots, S^{M}\right\}$ containing $M \mathrm{Z}-\mathrm{CS}_{P}^{N}\left\{S_{1}^{i}, S_{2}^{i}, \ldots, S_{P}^{i}\right\}(i=1,2 \ldots M)$ sets which are mutual mates. Without loss of generality, we assume $P=2$, $M=2$. Let $H$ denote a second-order Hadamard matrix

$$
H=\left[\begin{array}{l}
h_{1} \\
h_{2}
\end{array}\right]=\left[\begin{array}{ll}
h_{1}(0) & h_{1}(1) \\
h_{2}(0) & h_{2}(1)
\end{array}\right]=\left[\begin{array}{cc}
1 & 1 \\
1 & -1
\end{array}\right] .
$$

Let $\otimes$ denote Kronecker product operation, we have sequences of length $2 N P$ as (6). It can be proved that the even shifted ACF sidelobes and CCFs of $C_{1}$ and $C_{2}$ are zeros when $0<\tau<2 Z$. For any even time shift $\tau, 0<\tau<2 Z$, we always have (7) and (8).
Therefore, both the aperiodic and periodic even shifted CCFs of GESO-like sequences are zeros when we see (6)-(8) at the bottom of the page, where $0<\tau<2 Z$. Similarly, one can also show that both the aperiodic and periodic even shifted ACF sidelobes of GESO-like sequences are zeros when $0<\tau<2 Z$.

\section{WALSh-HADAMARD MATRIX EXPANSION AND ODD SHIFT CORRELATION ELIMINATION}

The number of GESO-like sequences generated above is small relative to its processing gain (PG). To overcome this problem, one may use Walsh-Hadamard matrix expansion as follows. Let $\mathrm{GC}_{1}=C$, and in general, we have

$$
G C_{K}=\left[\begin{array}{l}
G C_{1, K} \\
G C_{2, K}
\end{array}\right]=\left[\begin{array}{cc}
G C_{1, K / 2} & G C_{1, K / 2} \\
G C_{1, K / 2} & -G C_{1, K / 2} \\
G C_{2, K / 2} & G C_{2, K / 2} \\
G C_{2, K / 2} & -G C_{2, K / 2}
\end{array}\right]
$$

where each row will be viewed as a sequence and $K=2^{k}(k$ is a nonnegative integer).

It can be shown that the even shifted CCFs are always zero when the phase shift is smaller than $2 Z$. It is obviously that there are $M K$ sequences each of length $4 N K$.

The odd shift correlation elimination algorithm [6] will be used to remove all odd shift correlation levels of the sequences generated in the previous section. Let us define

$$
G C_{K}=\left[\begin{array}{llll}
g_{1} & g_{2} & \cdots & g_{M K}
\end{array}\right]^{T} .
$$

Based on $\mathrm{GC}_{K}$ and $H$, we can proceed to generate two new sequence sets

$$
\begin{aligned}
U_{I}(p, q) & =\left[\begin{array}{llll}
u_{I, 1} & u_{I, 2} & \cdots & u_{I, M K}
\end{array}\right]^{T} \\
& =\left[\begin{array}{llll}
g_{1} h_{1}(\zeta) & g_{2} h_{1}(\zeta) & \cdots & g_{M K} h_{1}(\zeta)
\end{array}\right]^{T} \\
U_{Q}(p, q) & =\left[\begin{array}{llll}
u_{Q, 1} & u_{Q, 2} & \cdots & u_{Q, M K}
\end{array}\right]^{T} \\
& =\left[\begin{array}{llll}
g_{1} h_{2}(\zeta) & g_{2} h_{2}(\zeta) & \cdots & g_{M K} h_{2}(\zeta)
\end{array}\right]^{T}(11)
\end{aligned}
$$

$$
\begin{aligned}
C= & {\left[\begin{array}{l}
C_{1} \\
C_{2}
\end{array}\right]=\left[\begin{array}{l}
S_{1}^{1} \otimes h_{1}, S_{2}^{1} \otimes h_{2} \\
S_{1}^{2} \otimes h_{1}, S_{2}^{2} \otimes h_{2}
\end{array}\right] } \\
= & {\left[\begin{array}{l}
S_{1}^{1}(0) h_{1}, \ldots, S_{1}^{1}(N-1) h_{1}, S_{2}^{1}(0) h_{2}, \ldots, S_{2}^{1}(N-1) h_{2} \\
S_{1}^{2}(0) h_{1}, \ldots, S_{1}^{2}(N-1) h_{1}, S_{2}^{2}(0) h_{2}, \ldots, S_{2}^{2}(N-1) h_{2}
\end{array}\right] } \\
P_{c_{1}, c_{2}(\tau)}= & 2\left[A_{S_{1}^{1}, S_{1}^{2}}\left(\frac{\tau}{2}\right)+A_{S_{2}^{1}, S_{2}^{2}}\left(\frac{\tau}{2}\right)\right]+\left[h_{1}(0) h_{2}(0)+h_{1}(1) h_{2}(1)\right] \\
& \cdot \sum_{i=0}^{\tau / 2-1}\left[S_{2}^{2}(i) S_{1}^{1}(N-1-i)+S_{1}^{2}(N-1-i)\right]=0 \\
A_{c_{1}, C_{2}}(\tau)= & 2\left[A_{S_{1}^{1}, S_{1}^{2}}\left(\frac{\tau}{2}\right)+A_{S_{2}^{1}, S_{2}^{2}}\left(\frac{\tau}{2}\right)\right]+\left[h_{1}(0) h_{2}(0)+h_{1}(1) h_{2}(1)\right] \\
& \cdot \sum_{i=0}^{\tau / 2-1}\left[S_{2}^{2}(i) S_{1}^{1}(N-1-i)\right]=0
\end{aligned}
$$


where $\zeta=(\bmod 2)$. Thus, we can combine (11) to form a complex sequences set $U$ as

$$
\begin{aligned}
U= & {\left[\begin{array}{llll}
u_{1} & u_{2} & \cdots & u_{M K}
\end{array}\right]^{T} } \\
= & {\left[\begin{array}{llll}
u_{I, 1} & u_{I, 2} & \cdots & u_{I, M K}
\end{array}\right]^{T} } \\
& +j\left[\begin{array}{llll}
u_{Q, 1} & u_{Q, 2} & \cdots & u_{Q, M K}
\end{array}\right]^{T}
\end{aligned}
$$

where $j$ is an imaginary unit.

The codes given in (12) are the desired GPZ codes. It can be seen from (12) that the obtained GPZ codes work in pairs as in-phase and quadrature components, respectively. Therefore, they can be sent via orthogonal carriers in a QPSK modulator. The PG of the GPZ codes is $4 N K$ with its number being $M K$.

\section{A. Example}

Let $S^{1}=\left\{S_{1}^{1}, S_{2}^{1}\right\}, S_{1}^{1}=[+++], S_{2}^{1}=[+-+], S^{2}=$ $\left\{S_{1}^{2}, S_{2}^{2}\right\}$ and $S_{1}^{2}=[+-+], S_{2}^{2}=[---]$, based on (6), we obtain

$$
\begin{aligned}
C & =\left[\begin{array}{l}
C_{1} \\
C_{2}
\end{array}\right] \\
& =\left[\begin{array}{l}
++++++++--,+--++-+--+ \\
++----++--,-++-+-+-+-
\end{array}\right] .
\end{aligned}
$$

It is easy to prove that the sequence set $C$ is a GESO-like sequence set.

To increase the family number of C, Walsh-Hadamard expansion is operated. Assumed that the Walsh-Hadamard expansion factor $K$ is 2, and $G C_{1}=C$, according to (9), (11), and (12), the desired GPZ codes $U$ can be generated as (13) at the bottom of the page. $U$ is a $Z C Z(40,4,6)$ sequence set, which concludes four sequences each of length 40 , the zero correlation zone is 6 .

\section{Periodic ANd APERIOdic CORRELATION PROPERTIES OF GPZ CODES}

Assume $u_{I, k}, u_{Q, k}$ and $u_{I, m}, u_{Q, m}$ are two different GPZ codes with length of $L$. The aperiodic ACFs and CCFs of the GPZ codes can be obtained as (14) and (15), respectively, where $G C_{K}(k, i)$ and $G C_{K}^{*}(k, i)$ denote the value and the conjugate value of the $k$ th row $i$ th column element of $G C_{K}$, respectively. If $\tau$ is an odd integer, we always have $\zeta_{1} \neq \zeta_{2}$ and

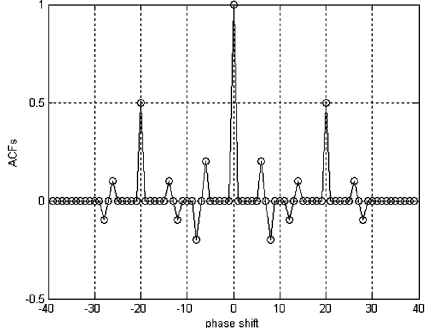

(a)

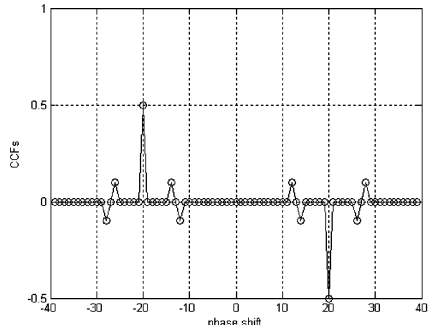

(b)
Fig. 1. Aperiodic correlation properties of GPZ codes $U$. (a) Aperiodic autocorrelation properties of $u_{1}$. (b) Aperiodic crosscorrelation properties of $u_{1}$ and $u_{2}$.

$h_{1}\left(\zeta_{1}\right) h_{1}\left(\zeta_{2}\right)+h_{2}\left(\zeta_{1}\right) h_{2}\left(\zeta_{2}\right)=0$; if $\tau$ is an even integer, we always have $\zeta_{1}=\zeta_{2}$ and $h_{1}\left(\zeta_{1}\right) h_{1}\left(\zeta_{2}\right)+h_{2}\left(\zeta_{1}\right) h_{2}\left(\zeta_{2}\right)=2$. However, since the correlation properties of GESO-like sequences, the correlation values are zero when $0 \leq \tau<2 Z$.

Fig. 1 shows the aperiodic ACFs and CCFs of a sample GPZ code set given in (13) generated by the approach given in this letter.

It is easy to show that the periodic ACF sidelobes and CCFs of the GPZ codes are zero when $0<\tau<2 Z$ by using the similar analysis as follows:

$$
\begin{aligned}
& \frac{1}{2}[\left.A_{u_{I, k}, u_{I, m}}(\tau)+A_{u_{Q, k}, u_{Q, m}}(\tau)\right] \\
&= \frac{1}{2}\left[\frac{1}{L} \sum_{i=0}^{L-1-\tau} u_{I}(k, i) u_{I}^{*}(m, i+\tau)\right. \\
&\left.\quad+\frac{1}{L} \sum_{i=0}^{L-1-\tau} u_{Q}(k, i) u_{Q}^{*}(m, i+\tau)\right] \\
&= \frac{1}{2} \frac{1}{L} \sum_{i=0}^{L-1-\tau}\left[G C_{K}(k, i) G C_{K}^{*}(m, i+\tau)\right] \\
& \times\left[h_{1}\left(\zeta_{1}\right) h_{1}\left(\zeta_{2}\right)+h_{2}\left(\zeta_{1}\right) h_{2}\left(\zeta_{2}\right)\right] \\
&= \begin{cases}0, & 0 \leq \tau<2 Z \\
\text { others, } & \text { else }\end{cases} \\
& \frac{1}{2}\left[A _ { u _ { I , K } , u _ { I , k } ( \tau ) + A _ { u _ { Q , K } , u _ { Q , K } } ( \tau ) ] } \left(\begin{array}{ll}
1, & \tau=0 \\
0, & 0<\tau<2 Z \\
\text { others }, & \text { else. }
\end{array}\right.\right.
\end{aligned}
$$

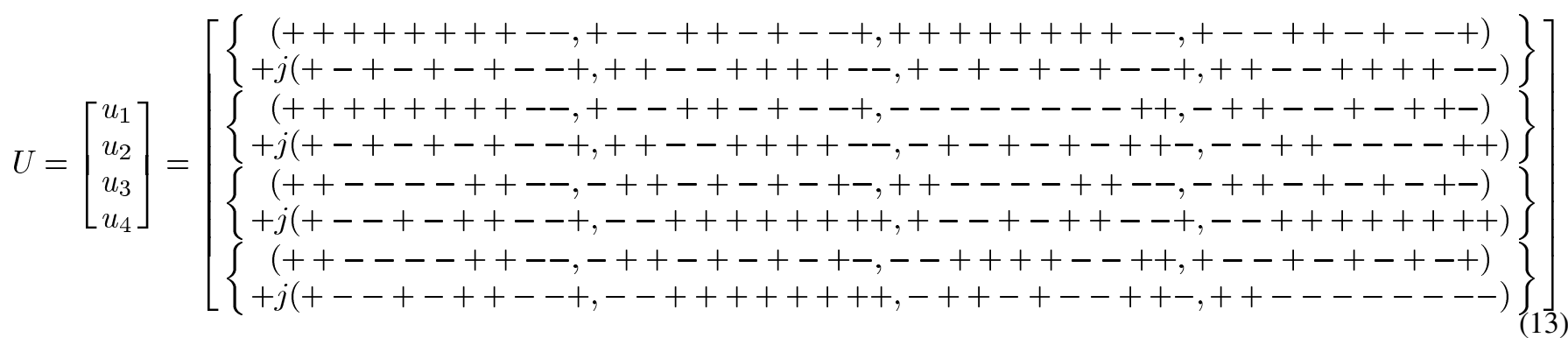


TABLE I

PARAMETERS OF GPC CODES AND GPZ CODES

\begin{tabular}{lll}
\hline Codes & GPC codes & GPZ codes \\
\hline $\begin{array}{l}\text { Length of } \\
\text { codes }\end{array}$ & $4 N K$ & $4 N K$ \\
\hline $\begin{array}{l}\text { Number } \\
\text { of codes }\end{array}$ & $2 K$ & $M K$ \\
\hline ZCZ & $8 N$ & $4 Z$ \\
\hline Remarks & $N, M$ respectively denote the length, number \\
& $\begin{array}{l}\text { of mates of both complete complementary } \\
\text { codes and Z-complementary codes; } Z \text { denotes } \\
\text { the zero correlation zone of Z-complementary } \\
\text { codes; } K \text { is a factor to perform } \\
\text { Walsh-Hadamard expansions. }\end{array}$ \\
\hline
\end{tabular}

Based on above analysis, we can describe the parameters of GPC codes and GPZ codes in Table I. It can be seen from Table I that the length of GPC codes are more limited than that of GPZ codes due to the fact that the length of complete complementary codes is limited by the allowable length $2^{a} 10^{b} 26^{c}$, where $a, b$, and $c$ are nonnegative integers while the length of Z-complementary codes can be an arbitrary integer bigger than 1 . Furthermore, the number of GPC codes is less than that of GPZ due to the fact that the number of complete complementary mates is limited by the number of codes while the number of Z-complementary mates can be bigger than 2 . As the cost, the zero correlation zone of GPZ codes is smaller than that of GPC codes.

\section{CONCLUSION}

In this letter, a general construction of OVSF codes with $\mathrm{ZCZ}$ property is proposed based on orthogonal sequence set and Z-complementary sets. It is shown that the proposed codes include the conventional OVSF codes and the ternary OVSF codes with ZCZ property constructed by binary complementary sets as special cases, and the proposed codes can accommodate more flexible rate assignment.

\section{REFERENCES}

[1] F. Adachi, M. Sawahashi, and K. Okawa, "Tree-structured generation of orthogonal spreading codes with different lengths for forward link of DS-CDMA mobile radio," Electron. Lett., vol. 33, pp. 27-28, Jan. 1997.

[2] E. Dahlman, B. Gudmundson, M. Nilsson, and J. Skold, "UMTS/IMT2000 based on wideband CDMA," IEEE Commun. Mag., vol. 36, no. 9, pp. 70-80, Sep. 1998.

[3] C. M. Yang, P. H. Lin, G. C. Yang, and W. C. Kwong, "2D orthogonal spreading codes for multicarrier DS-CDMA systems," in Proc. IEEE Int. Conf. Communications, ICC'03, May 11-15, 2003, vol. 5, pp. 3277-3281.

[4] D. Wu, P. Spasojević, and I. Seskar, "Ternary complementary sets for orthogonal pulse based UWB," in Proc. Conf. Record 37th Asilomar Conf. Signals, Systems and Computers, Nov. 2003, vol. 2, pp. 1776-1780.

[5] P. Z. Fan, N. Suehiro, N. Kuroyanagi, and X. M. Deng, "Class of binary sequences with zero correlation zone," Electron. Lett., vol. 35, pp. 777-779, May 1999.

[6] D. Wu, P. Spasojević, and Seskar, "Orthogonal variable spreading factor codes with zero-correlation zone for TS-UWB," in Proc. IEEE Wireless Communications and Networking Conf., WCNC 2, 2005, pp. 807-812.

[7] D. Wu, P. Spasojević, and I. Seskar, "Ternary zero-correlation zone sequences for multiple code UWB," in Proc. 38th Conf. Information Sciences and Systems, Princeton, NJ, Mar. 17-19, 2004, pp. 939-943.

[8] D. Wu and P. Spasojevic, "Adaptive rate QS-CDMA UWB systems using ternary OVSF codes with a zero-correlation zone," in Proc. IEEE Wireless Communications and Networking Conf., Apr. 3-6, 2006, vol. 2, pp. 1068-1073.

[9] A. Gavish and A. Lempel, "On ternary complementary sequences," IEEE Trans. Inf. Theory, vol. 40, no. 3, pp. 522-526, Mar. 1994.

[10] P. Z. Fan, W. N. Yuan, and Y. F. Tu, "Z-complementary binary sequences," IEEE Signal Process. Lett., vol. 14, no. 6, pp. 401-404, Jun. 2007. 